\title{
Relationship between Perioperative N-Terminal Pro-Brain Natriuretic Peptide (NT-proBNP) and Complications in Thoracic Surgery
}

\author{
Kantaro Hara, Takuma Tsukioka*, Nobuhiro Izumi, Kyukwang Chung, Hiroaki Komatsu, \\ Michihito Toda, Hikaru Miyamoto, Satoshi Suzuki, Takuya Kimura, Toshihiko Shibata, \\ Noritoshi Nishiyama
}

Department of Thoracic Surgery, Osaka City University Hospital, Osaka, Japan

Email: *m1156870@med.osaka-cu.ac.jp

How to cite this paper: Hara, K., Tsukioka, T., Izumi, N., Chungm K., Komatsu, H., Toda, M., Miyamoto, H., Suzuki, S., Kimura, T., Shibata, T. and Nishiyama, N. (2018) Relationship between Perioperative N-Terminal Pro-Brain Natriuretic Peptide (NT-proBNP) and Complications in Thoracic Surgery. Open Journal of Thoracic Surgery, 8, 29-36.

https://doi.org/10.4236/ojts.2018.82006

Received: May 10, 2018

Accepted: June 11, 2018

Published: June 14, 2018

Copyright (c) 2018 by authors and Scientific Research Publishing Inc. This work is licensed under the Creative Commons Attribution International License (CC BY 4.0).

http://creativecommons.org/licenses/by/4.0/

\begin{abstract}
Background: In cardiac surgery, the $\mathrm{N}$-terminal pro-brain natriuretic peptide (NT-proBNP) level is reportedly correlated with the onset of postoperative complications. We examined the relationship between the NT-proBNP level and perioperative complications in thoracic surgery and elucidated the significance of NT-proBNP measurement. Methods: We evaluated 48 patients excluding hemodialysis patients who underwent lung resection from November 2015 to February 2016. NT-proBNP measurement was performed three times preoperatively on postoperative days 2 and 5 . We examined the relationship between clinical background factors such as preoperative comorbidity and the development of postoperative complications. Based on established criteria for surgical complications (Clavien-Dindo classification), grade $\geq$ II was defined as a complications. We classified the patients into two groups (with or without complications) and compared and examined the background factors between the groups. Results: The study included 48 patients ( 33 male, 15 female) with an average age of 72 years (range, 35 - 86 years). Preoperative comorbidities included cardiovascular disease in five patients, diabetes mellitus in four, and chronic obstructive pulmonary disease in six. The average operation time was 163 (29 - 308) minutes, and the bleeding volume was $30(10-620)$ ml. Early complications were confirmed in 11 patients, and late complications were confirmed in 7 patients. A high NT-proBNP level on postoperative day 5 was an independent risk factor for late complications. Conclusion: The perioperative NT-proBNP level is considered to be a predictor of complications. Its measurement is useful for predicting postoperative complications, and careful pe-
\end{abstract}


rioperative management is required for patients with high levels.

\section{Keywords}

NT-proBNP, Perioperative Management, Lung Resection, Postoperative Complications

\section{Introduction}

The prediction of postoperative complication is important for perioperative management and it is also a factor in determining surgical indication. $\mathrm{N}$-terminal fragment Brain Natriuretic Peptide (NT-proBNP) is thought to be an indicator of heart failure similarly to BNP [1]. In the thoracic surgery, the preoperative NT-proBNP is said to be an early complication predictor [2]. On the other hand, prediction of late complications is difficult. We examined how the NT-proBNP level changes during the perioperative period of thoracic surgery and evaluated it as a predictor of postoperative complications.

\section{Materials and Methods}

\subsection{Patients}

Of 63 patients who underwent lung resection for lung nodule at our hospital from November 2015 to February 2016, we retrospectively studied consecutive 48 patients who NT-pro BNP was measured three times during the perioperative period. Patients who were undergoing hemodialysis, or were unable to complete the measurement of NT-proBNP were excluded. The comorbidity status was evaluated objectively using the Simplified Comorbidity Score (SCS) [3], which is a partially modified composite of comorbidity. Blood samples were taken from each patient within two weeks before surgery. C-reactive protein (CRP) and serum albumin were measured. Glasgow Prognostic score (GPS) was analysed. GPS consisted of the combination of CRP and albumin measurements. Patients with normal CRP $(\leq 1.0 \mathrm{mg} / \mathrm{dl})$ and normal albumin $(\geq 3.5 \mathrm{~g} / \mathrm{dl})$ were classified in GPS 0. Patients with either one abnormal factor were classified in GPS 1. Those with both abnormal factors were classified in GPS 2. Informed consent was obtained from all the eligible patients prior to inclusion. The study protocol was approved by the local regional ethics committee.

\subsection{Postoperative Complications}

Each postoperative complication was accurately assessed and graded according to the Clavien-Dindo classification, which is based on the treatment required to treat any given postoperative complications [4] [5]. We defined complications during hospitalization as "early complications". "Late complication" was defined as complications that required treatment occurred within three months after discharge. Postoperative pain was excluded. 


\subsection{Measuring Serum NT-proBNP}

All of analytical measurements were performed at the Central Laboratory of the Osaka City University Hospital. Fasting hematological, biochemical profile and the levels of NT-proBNP were collected and measured before surgery, second and fifth postoperative day.

\subsection{Statistical Analysis}

Continuous variables were presented as median with SD depending on the normality of the distribution and compared using Student's t-test, whereas categorical variables were given as frequencies with percentages and compared with $\chi^{2}$ test. The cut-off value of NT-proBNP was calculated according for Receiver operating characteristic (ROC) curves which were applied to obtain the area under the curve (AUC) and to determine ideal cut-offs. The cut-off values of age, hemoglobin, eGFR, blood loss, and time of operation were calculated according for medians in this study. Logistic regression analysis was applied to identify independent predictors; variables with $p$ value $<0.1$ in univariable analyses were further included in multivariable analyses. A $p$ value $<0.05$ was considered to be statistically significant in all tests. Data analyses were generated using JMP ${ }^{\bowtie} 10$ (SAS Institute Inc., Cary, NC, USA).

\section{Results}

Forty eight patients comprised 33 men and 15 women, with median age at the time of surgery of 72 years (range 35 - 86 years). The following types of lung resection were performed: lobectomy (32; 66.7\%), segmentectomy and wedge resection $(16 ; 33.3 \%)$. Early complications developed in twelve patients and late complications developed in seven patients (Table 1). The clinical background of patients with and without late complications is shown in Table 2. In the group with late complications, perioperative NT-proBNP level was significantly higher than in the group without those. The areas under the ROC curve, which signifies the utility of postoperative fifth day's NT-proBNP level in predicting late complication, was 0.83 (Figure 1). The cut-off values of NT-proBNP levels in predicting the development of late complication were $396 \mathrm{pg} / \mathrm{ml}$. Similarly, the areas under the ROC curve, which signifies the utility of postoperative second day's NT-proBNP level in predicting late complication, was 0.72 . The cut-off value of NT-proBNP levels in predicting the development of late complication was 573 $\mathrm{pg} / \mathrm{ml}$. We then performed statistical analysis on late complications. In the group with late complications, postoperative fifth day's NT-proBNP level was significantly higher than in the group without those. The univariate predictors for late complications included diabetes, development of early complications, postoperative second day's NT-proBNP level and postoperative fifth day's NT-proBNP level. In the multivariate analysis, only postoperative fifth day's NT-proBNP level remained significant as a predictor for late complications (Table 3). 


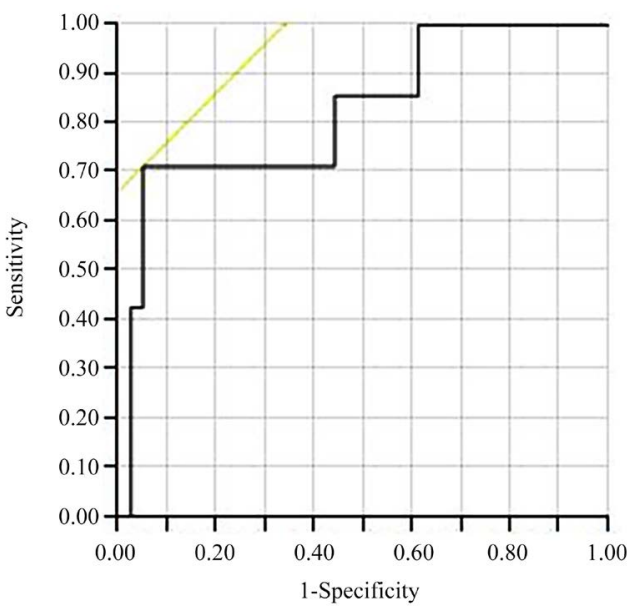

Figure 1. ROC curve for development of late complications according to different cut-off values of postoperative fifth day's NT-proBNP level. Optimal cut off level was $396 \mathrm{pg} / \mathrm{ml}$ and area under the curve: 0.83 .

Table 1. Early and late complications.

\begin{tabular}{ccc}
\hline \multirow{2}{*}{ Variable } & \multicolumn{2}{c}{ Number of patients $(\mathrm{N}=48, \%)$} \\
\cline { 2 - 3 } All complications & Early complications & Late complications \\
\hline Prolonged air leakage & $11(23 \%)$ & $7(15 \%)$ \\
Pneumonia & 4 & 2 \\
Atelectasis & 3 & 0 \\
Interstitial pneumonia & 1 & 1 \\
Chylothorax & 1 & 0 \\
Atrial fibrillation & 1 & 3 \\
Abnormality of coagulation & 2 & 1 \\
\hline
\end{tabular}

Table 2. Clinical and demographic characteristics of patients with or without late complications.

\begin{tabular}{cccc}
\hline \multirow{2}{*}{ Characteristics } & With late complications & Without late complications & \multirow{2}{*}{$p$ value } \\
\cline { 2 - 3 } Gender Male/Female & $\mathrm{n}=7$ & $\mathrm{n}=41$ & 0.295 \\
Age Median (range year) & $62(70-85)$ & $72(35-86)$ & 0.186 \\
Smoking & $1 / 6$ & $16 / 25$ & 0.206 \\
Diabetes & 2 & 2 & 0.036 \\
Cardiovascular comorbidity & 2 & 5 & 0.089 \\
\% DLco, \% median & $85(53.6-157)$ & $68(55-360)$ & 0.485 \\
LVEF, \% median & $60(60-65)$ & $60(44-63)$ & 0.101 \\
$\begin{array}{c}\text { Preoperative Hb }(\mathrm{g} / \mathrm{dl}) \\
\text { Preoperative eGFR } \\
\left.\text { (mL/min/1.73 m }{ }^{2}\right)\end{array}$ & $11.6(10.0-16.7)$ & $13.4(9.5-18.6)$ & 0.073 \\
GPS $0 / 1 / 2$ & $64.7(50.0-105.6)$ & $72.3(47.7-98.4)$ & 0.864
\end{tabular}




\section{Continued}

\begin{tabular}{|c|c|c|c|}
\hline SCS & $8(5-13)$ & $7(0-13)$ & 0.146 \\
\hline PS $0 / 1 / 2$ & $6 / 1 / 0$ & $40 / 1 / 0$ & 0.154 \\
\hline $\begin{array}{l}\text { Type of operation } \\
\text { Lobectomy }\end{array}$ & 3 & 29 & 0.160 \\
\hline Others & 4 & 12 & \\
\hline Blood loss $(\mathrm{mL})$, median & $30(10-400)$ & $20(10-620)$ & 0.411 \\
\hline $\begin{array}{l}\text { Time of operation }(\mathrm{min}), \\
\text { median }\end{array}$ & $173(110-308)$ & $153(29-284)$ & 0.158 \\
\hline Diagnosis Lung cancer & 6 & 33 & 0.743 \\
\hline Metastatic cancer & 1 & 6 & \\
\hline Others & 0 & 2 & \\
\hline $\begin{array}{l}\text { NT-proBNP admission } \\
(\mathrm{pg} / \mathrm{ml})\end{array}$ & $338(21-420)$ & $76(8-1275)$ & 0.134 \\
\hline 2nd operative days & $573(47-1725)$ & $154(14-1724)$ & 0.004 \\
\hline 5th operative days & $527(79-1128)$ & $103(12-1235)$ & $<0.001$ \\
\hline Early complications & 4 & 7 & 0.031 \\
\hline
\end{tabular}

\#DLco: diffusing capacity of the lung for carbon monoxide, LVEF: left ventricular ejection fraction, GPS: Glasgow Prognosis Scale, SCS: Simplified Comorbidity Score, PS: performance status\# values are median (range).

Table 3. Univariate and multivariate analysis for predictors of late complications.

\begin{tabular}{|c|c|c|c|c|c|c|}
\hline \multirow{2}{*}{ Variables } & \multicolumn{3}{|c|}{ Univariate } & \multicolumn{3}{|c|}{ Multivariate } \\
\hline & HR & $95 \% \mathrm{CI}$ & $p$ value & HR & $95 \% \mathrm{CI}$ & $p$ value \\
\hline Gender (Male) & 3.11 & $0.47-61.8$ & 0.266 & & & \\
\hline Age $(>72)$ & 2.16 & $0.41-16.3$ & 0.372 & & & \\
\hline Smorking $(\mathrm{BI}>200)$ & 3.83 & $0.58-76.1$ & 0.178 & & & \\
\hline Diabetes $(+)$ & 7.80 & $0.80-78.6$ & 0.075 & 28.2 & $0.87-1616$ & 0.059 \\
\hline Cardiovascular comorbidity $(+)$ & 5.07 & $0.57-39.0$ & 0.135 & & & \\
\hline$\%$ DLco $<70 \%$ & 1.03 & $0.14-21.2$ & 0.980 & & & \\
\hline Preoperative $\mathrm{Hb}(<13.3 \mathrm{~g} / \mathrm{dl})$ & 3.19 & $0.61-24.2$ & 0.173 & & & \\
\hline $\begin{array}{c}\text { Preoperative eGFR } \\
\left(<70.8 \mathrm{ml} / \mathrm{min} / 1.73 \mathrm{~m}^{2}\right)\end{array}$ & 3.19 & $0.61-24.2$ & 0.173 & & & \\
\hline GPS $\geq 2$ & 2.11 & $0.09-20.0$ & 0.566 & & & \\
\hline SCS & 1.20 & $0.62-1.04$ & 0.117 & & & \\
\hline $\mathrm{PS} \geq 1$ & 6.67 & $0.01-4.12$ & 0.223 & & & \\
\hline $\begin{array}{l}\text { Type of operation } \\
\text { (Lobectomy vs Others) }\end{array}$ & 3.22 & $0.62-18.5$ & 0.160 & & & \\
\hline Blood loss $(>30 \mathrm{ml})$ & 1.06 & $0.19-5.41$ & 0.945 & & & \\
\hline Time of operation (>163 min) & 2.89 & $0.55-21.9$ & 0.213 & & & \\
\hline Early complication & 6.48 & $1.19-39.7$ & 0.031 & 2.36 & $0.19-30.9$ & 0.489 \\
\hline $\begin{array}{l}\text { Postoperative second day NT-proBNP } \\
\text { level }>573 \mathrm{pg} / \mathrm{ml}\end{array}$ & 16.9 & $2.66-133$ & 0.003 & 7.76 & $0.46-215$ & 0.152 \\
\hline $\begin{array}{c}\text { Postoperative fifth day NT-proBNP } \\
\text { level }>396 \mathrm{pg} / \mathrm{ml}\end{array}$ & 48.8 & $6.65-583$ & $<0.001$ & 41.7 & $3.39-1315$ & 0.003 \\
\hline
\end{tabular}




\section{Discussion}

Regarding the early complications, in our study, the BNP level was significantly higher in the early complications group (Figure 2). This result was consistent with other literatures [2] [6] [7]. Also, this study shows that postoperative fifthday's NT-proBNP level became a risk factor for the onset of late complications after discharge in case with high levels. Using the ROC curve, we leaded that postoperative fifth day's NT-proBNP level of $396 \mathrm{pg} / \mathrm{ml}$ was optimal cutoff point for prediction of late complications. Among 11 cases of early complications, late complications also occurred in $4(36.3 \%)$ cases. In other words, late complications were not observed in the remaining 7 cases. We investigated the four cases in detail. One patient experienced atrial fibrillation on the 3 rd postoperative day and relieved with internal medicine, but after discharge she had coagulation abnormality and internal medication for anticoagulant was needed. Two patients showed postoperative prolonged air leakage. Of the two, one patient developed pneumonia after discharge and died. The other patient was admitted with atrial fibrillation in the first month after discharge. In the last one patient, both early and late complications were exacerbations of interstitial pneumonia. In the last case of interstitial pneumonia, late complications were thought to relapse of early complications. Furthermore, in multivariate analysis, the factor of postoperative fifth day's NT-proBNP level was an independent factor than the factor of early complications.

Production of BNP and NT-proBNP is derived from the same BNP gene. From the BNP gene, BNP precursor is generated after transcription/translation, and then it is cleaved into physiologically inactive NT-proBNP and mature BNP with physiological activity. BNP and NT-proBNP are mainly generated in the ventricle, gene expression is enhanced according to extension stress, and it is rapidly generated and secreted [8]. Therefore, in heart failure with increased

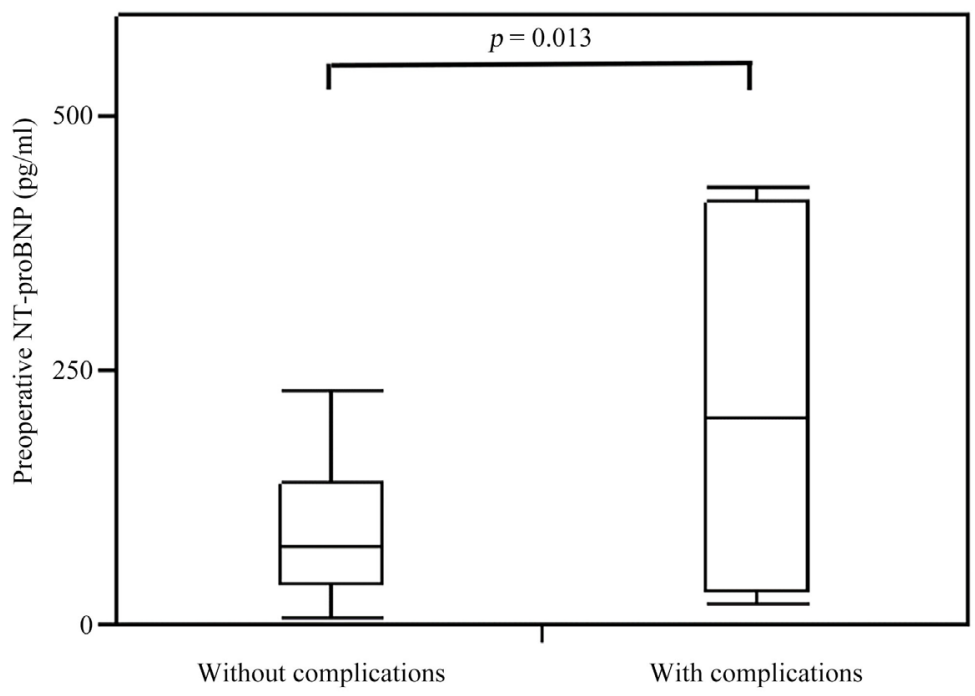

Figure 2. Comparison of preoperative NT-proBNP measurements between patients with early complications and without those. 
stretching stress, its blood level increases according to its severity. Besides stress to the cardiac muscle, there are factors that affect blood concentrations of both peptides. In particular, most of its metabolism of NT-proBNP depends on excretion by filtration from the kidney. Even mild renal function decline is affected, and cases of eGFR less than $30 \mathrm{ml} / \mathrm{min} / 1.73 \mathrm{~m}^{2}$ increase the degree of increase. Blood concentrations also increase in elderly people. In addition, acute inflammation may show high level. Conversely, in obese people both peptides show lower level than non obese people. There is almost no report on the transition of BNP and NT-proBNP level in the perioperative period of non-cardiac surgery. Hou reported both high NT-proBNP level immediately after surgery and high preoperative NT-proBNP level were related to the appearance of postoperative atrial fibrillation with esophageal cancer surgery [9]. Nojiri reported that the increase in the BNP level at the perioperative period was significantly related to postoperative atrial fibrillation after lung resection [2] [6] [7]. It has also been reported that increased BNP level is associated with pulmonary diseases including not only primary pulmonary hypertension but also COPD [10] [11]. We believe NT-proBNP level encompasses backgrounds that can be risk factors for postoperative complications such as aging, anemia, renal dysfunction and cardiopulmonary comorbidity. In this study, as seen in other literature, the preoperative NT-proBNP high level became the predictive factor of the early complications. Furthermore, we found a method that can select cases requiring careful follow-up observation even after discharge by measuring NT-proBNP even after surgery.

The major limitations associated with the present study are small sample size and its retrospective and single-institution design. Thus, selection bias may exist. However, in the case of perioperative NT-proBNP high group, we should be cautious with the onset of early and late complications.

In conclusion, in thoracic surgery, the preoperative NT-proBNP high case was a risk factor for the onset of postoperative complications. NT-proBNP level changes greatly after surgery, but in cases with prolonged high level, risk of postoperative and late complications is considered to increase. Measurement of NT-proBNP in the perioperative period is useful for predicting perioperative complications.

\section{References}

[1] Hunt, P.J., Richards, A.M., Nicholls, M.G., Yandle, T.G., Doughty, R.N. and Espiner, E.A. (1997) Immunoreactive Amino-Terminal Pro-Brain Natriuretic Peptide (NT-PROBNP): A New Marker of Cardiac Impairment. Clinical Endocrinology, 47, 287-296. https://doi.org/10.1046/j.1365-2265.1997.2361058.x

[2] Nojiri, T., Inoue, M., Takeuchi, Y., Maeda, H., Shintani, Y., Sawabata, N., et al. (2015) Impact of Cardiopulmonary Complications of Lung Cancer Surgery on Long-Term Outcomes. Surgery Today, 45, 740-745. https://doi.org/10.1007/s00595-014-1032-z

[3] Colinet, B., Jacot, W., Bertrand, D., Lacombe, S., Bozonnat, M.C., Daurès, J.P., et al. 
(2005) A New Simplified Comorbidity Score as a Prognostic Factor in Non-Small-Cell Lung Cancer Patients: Description and Comparison with the Charlson's Index. British Journal of Cancer, 93, 1098-1105. https://doi.org/10.1038/sj.bjc.6602836

[4] Dindo, D., Demartines, N. and Clavien, P.A. (2004) Classification of Surgical Complications: A New Proposal with Evaluation in a Cohort of 6336 Patients and Results of a Survey. Annals of Surgery, 240, 205-213. https://doi.org/10.1097/01.sla.0000133083.54934.ae

[5] Clavien, P.A., Barkun, J., de Oliveira, M.L., Vauthey, J.N., Dindo, D., Schulick, R.D., et al. (2009) The Clavien-Dindo Classification of Surgical Complications: Five-Year Experience. Annals of Surgery, 250, 187-196.

https://doi.org/10.1097/SLA.0b013e3181b13ca2

[6] Nojiri, T., Maeda, H., Takeuchi, Y., Funakoshi, Y., Kimura, T., Maekura, R., et al. (2010) Predictive Value of B-Type Natriuretic Peptide for Postoperative Atrial Fibrillation Following Pulmonary Resection for Lung Cancer. European Journal of Cardio-Thoracic Surgery, 37, 787-791. https://doi.org/10.1016/j.ejcts.2009.09.043

[7] Nojiri, T., Inoue, M., Yamamoto, K., Maeda, H., Takeuchi, Y., Funakoshi Y., et al. (2011) B-Type Natriuretic Peptide as a Predictor of Postoperative Cardiopulmonary Complications in Elderly Patients Undergoing Pulmonary Resection for Lung Cancer. The Annals of Thoracic Surgery, 92, 1051-1055. https://doi.org/10.1016/j.athoracsur.2011.03.085

[8] Daniels, L.B. and Maisel, A.S. (2007) Natriuretic Peptides. Journal of the American College of Cardiology, 50, 2357-2368. https://doi.org/10.1016/j.jacc.2007.09.021

[9] Hou, J.L., Gao, K., Li, M., Ma, J.Y., Shi, Y.K., Wang, Y., et al. (2008) Increased N-Terminal Pro-Brain Natriuretic Peptide Level Predicts Atrial Fibrillation after Surgery for Esophageal Carcinoma. World Journal of Gastroenterology, 14, 2582-2585.

[10] Nagaya, N., Nishikimi, T., Okano, Y., Uematsu, M., Satoh, T., Kyotani, S., et al. (1998) Plasma Brain Natriuretic Peptide Levels Increase in Proportion to the Extent of Right Ventricular Dysfunction in Pulmonary Hypertension. Journal of the American College of Cardiology, 31, 202-208. https://doi.org/10.1016/S0735-1097(97)00452-X

[11] Inoue, Y., Kawayama, T., Iwanaga, T. and Aizawa, H. (2009) High Plasma Brain Natriuretic Peptide Levels in Stable COPD without Pulmonary Hypertension or Cor Pulmonale. Internal Medicine Journal, 48, 503-512. 